\title{
Artificial Lagrange Points for Solar Sail with Electrochromic Material Panels
}

\author{
Generoso Aliasi; $\quad$ Giovanni Mengali ${ }^{\dagger}$ and Alessandro A. Quarta ${ }^{\ddagger}$ \\ University of Pisa, I-56122 Pisa, Italy
}

\section{Introduction}

Due to its inherent capability of producing thrust without using any propellant consumption, a solar sail is a particularly attractive option for the generation of so-called Artificial Equilibrium Points (AEPs). These points are of great interest for mission applications because the spacecraft can be used to provide new vantage points for scientific observation [1]. Interesting positions for AEPs are those placed along the segment connecting the two primaries in the neighborhood of the classical $L_{1}$ Lagrange Point. For example, in the Sun-(Earth+Moon) system, these $L_{1}$-type AEPs have been suggested as useful locations for space weather observation missions [2] or for geo-engineering missions [3,4].

Because $L_{1}$-type AEPs are intrinsically unstable [5], a suitable control strategy is required to maintain their desired location. This problem has been addressed with different approaches, which mainly consider either a pitch and yaw-angle control, or a solar sail area variation [6]. However, both solutions present some drawbacks.

A substantial simplification of the control problem is obtained when the solar sail attitude is maintained fixed, in a passive way, using a conically shaped structure [7]. The propulsive thrust is therefore always in the Sun-spacecraft direction, but it can be modulated by varying, within a limited range, the ratio of the solar radiation pressure acceleration to the solar

*Ph.D. Candidate, Department of Aerospace Engineering, g.aliasi@dia.unipi.it.

${ }^{\dagger}$ Associate Professor, Department of Aerospace Engineering, g.mengali@ing.unipi.it. Senior Member AIAA.

${ }^{\ddagger}$ Research Assistant, Department of Aerospace Engineering, a.quarta@ing.unipi.it. Senior Member AIAA. 
gravitational acceleration, that is, the sail lightness number $\beta$. Such a solution is commonly referred to as $\beta$-control. The original idea of a $\beta$-control applied to a solar-sail-based mission towards a $L_{1}$-type AEP is due to Biggs and McInnes [4]. An interesting implementation of a $\beta$-control makes use of Electrochromic Material Panels (EMPs) [8]. These materials have been already employed in space missions, notably for the attitude control of the Japanese solar sail demonstrator IKAROS $[9,10]$.

The aim of this Note is to explore the capabilities of the emerging EMP technology for the active stabilization of $L_{1}$-type AEPs using a square solar sail with a fixed attitude. The problem is addressed within an elliptic restricted framework, which is a more realistic model with respect to the classical circular case [4]. The main spacecraft parameters, including the sail side and the total spacecraft mass, are defined, by means of a simplified mathematical model, as a function of the main mission requirements in terms of maximum allowed sail lightness number variation and AEP position.

\section{Mathematical Preliminaries}

Consider the motion of a solar sail within an elliptic restricted three-body problem (ERTBP) with the Earth and the Sun as the two primaries and the spacecraft as the (massless) third body. Both Sun and Earth rotate around their center of mass $C$ and cover elliptic orbits with the same eccentricity $e$, and semimajor axes $\mu a$ and $(1-\mu) a$, respectively, where $\mu \triangleq 1 / 328900.56$ is the Earth's dimensionless mass $[11,12]$. Introduce a non-uniformly rotating and pulsating reference frame $\mathcal{T}(C ; x / \ell, y / \ell, z / \ell)$, in which $\ell$ is the time-dependent distance between the two celestial bodies, the $(x, y)$ plane coincides with the plane of motion of the primaries, $x$ points toward the Earth at any instant of time and rotates around $z$ [11]. Note that $\ell=a\left(1-e^{2}\right) g$, where $g \triangleq 1 /(1+e \cos \nu)$ and $\nu$ is the Earth's true anomaly. Also, denote $\boldsymbol{r}, \boldsymbol{\rho}_{S}$ and $\boldsymbol{\rho}_{E}$ as the spacecraft's dimensionless position vectors (normalized by $\ell$ ) 
with respect to $C$, Sun, and Earth, respectively.

In the following the solar sail is assumed to provide a purely radial acceleration with respect to the Sun $[7,13]$. This situation is representative of a solar sail whose attitude is maintained in a passive way by means of a suitable design of the sail shape. The spacecraft propulsive acceleration can be written as

$$
\boldsymbol{a}_{P}=\beta \frac{\mu_{\odot}}{\ell^{2} \rho_{S}^{3}} \boldsymbol{\rho}_{S}
$$

where $\mu_{\odot} \triangleq 132712439935.5 \mathrm{~km}^{3} / \mathrm{s}^{2}$ is the Sun's gravitational parameter and $\rho_{S}=\left|\boldsymbol{\rho}_{S}\right|$.

The dimensionless vectorial equation of motion of the solar sail spacecraft in the rotating frame $\mathcal{T}$ is $[14]:$

$$
\boldsymbol{r}^{\prime \prime}+2 \hat{\boldsymbol{k}} \times \boldsymbol{r}^{\prime}=g\left[-\frac{(1-\beta)(1-\mu)}{\rho_{S}^{3}} \boldsymbol{\rho}_{S}-\frac{\mu}{\rho_{E}^{3}} \boldsymbol{\rho}_{E}-\hat{\boldsymbol{k}} \times(\hat{\boldsymbol{k}} \times \boldsymbol{r})-e \cos \nu(\boldsymbol{r} \cdot \hat{\boldsymbol{k}}) \hat{\boldsymbol{k}}\right]
$$

where the prime symbol denotes a derivative taken with respect to the angular coordinate $\nu$ and $\hat{\boldsymbol{k}}$ is the unit vector of $(z / \ell)$-axis. Let $P_{0}$ be a $L_{1}$-type AEP (with subscript 0 ), that is, a point located along the $(x / \ell)$-axis between the Sun and the classical Lagrange point $L_{1}$, where $\rho_{L_{1}} \simeq 0.989989$ is the Sun- $L_{1}$ dimensionless distance. It can be shown [14-16] that the sail lightness number $\beta_{0}$ required for placing $P_{0}$ at a given distance $\rho_{S_{0}} \in\left(0, \rho_{L_{1}}\right]$ from the Sun is

$$
\beta_{0}=1-\frac{\mu}{1-\mu}\left[\frac{\rho_{S_{0}}}{\mu}+\frac{1}{\left(1-\rho_{S_{0}}\right)^{2}}-1\right] \rho_{S_{0}}^{2}
$$

where $\rho_{S_{0}}=r_{0}+\mu$. Equation (2) is now linearized around $P_{0}$, where $\boldsymbol{r}_{0}^{\prime \prime}=\boldsymbol{r}_{0}^{\prime}=0$ by definition. Using the transformations $\boldsymbol{r}=\boldsymbol{r}_{0}+\delta \boldsymbol{r}$ and $\beta=\beta_{0}+\delta \beta$, with $|\delta \boldsymbol{r}| \ll\left|\boldsymbol{r}_{0}\right|$ and $\delta \beta \ll \beta_{0}$, and introducing the state vector $\boldsymbol{\xi} \triangleq\left[\delta \boldsymbol{r}^{\mathrm{T}}, \delta \boldsymbol{r}^{\prime \mathrm{T}}\right]^{\mathrm{T}}$, the linearized equation of motion 
can be written in matrix form as

$$
\boldsymbol{\xi}^{\prime}=\left[\begin{array}{cc}
0_{3 \times 3} & I_{3 \times 3} \\
g K & -2 E
\end{array}\right] \boldsymbol{\xi}+\left[\begin{array}{c}
0_{3 \times 1} \\
g U
\end{array}\right] \delta \beta
$$

where $I$ is the identity matrix and

$$
K \triangleq\left[\begin{array}{ccc}
-2 k_{22}+3 & 0 & 0 \\
0 & k_{22} & 0 \\
0 & 0 & k_{22}-1
\end{array}\right] \quad, \quad E \triangleq\left[\begin{array}{ccc}
0 & -1 & 0 \\
1 & 0 & 0 \\
0 & 0 & 0
\end{array}\right] \quad, \quad U \triangleq\left[\begin{array}{c}
\frac{1-\mu}{\rho_{S_{0}}^{2}} \\
0 \\
0
\end{array}\right]
$$

with $k_{22} \triangleq\left(\mu / \rho_{S_{0}}\right)\left[1-1 /\left(1-\rho_{S_{0}}\right)^{3}\right]$.

\section{The $\beta$-control Concept}

It is known that $L_{1}$-type AEPs are intrinsically unstable [5], and therefore a suitable control strategy is necessary to maintain their desired location [17]. Assuming that the sail pitch and yaw angles are not actively controlled $[18,19]$, an interesting solution for stabilizing a solar sail spacecraft about an $L_{1}$-type AEP, is to vary the sail lightness number in such a way that $\beta$ tracks a desired control law $[4,6]$.

A straightforward approach is to use a proportional control law, in the form $\delta \beta=-k \delta_{x}$, where $k>0$ is a pure gain, and $\delta_{x} \triangleq(x / \ell)-r_{0}$ is the component along the $x$-axis of the position error vector $\delta \boldsymbol{r}$. With such a simple control logic it may be shown [4] that there exists a minimum value of $k$, depending on the distance $\rho_{S_{0}}$, the eccentricity $e$ and the dimensionless mass $\mu$, beyond which the $L_{1}$-type AEP is stable. Here stability is intended in the sense of Lyapunov, because the spacecraft trajectory remains bounded, even if asymptotic stability is not possible [4].

A feasible solution is offered by a proportional-derivative (PD) control logic, which has 
been shown to guarantee an asymptotical stability, in the $(x, y)$ plane, for the spacecraft trajectory [4]. The out of plane dynamics, instead, cannot be driven to converge toward the AEP because the motion along $(z / \ell)$-axis is not controllable but only $\beta$-stabilizable (in the sense of Lyapunov) about the given AEP.

A PD control logic, however, could produce unacceptable errors in the final spacecraft position when an uncertainty in the actual sail lightness number occurs. Indeed, if the actual value of the sail lightness number is (slightly) different from the nominal value given by Eq. (3), the spacecraft trajectory converges to an AEP different from the desired one.

To get over this problem, a classical Proportional-Integral-Derivative (PID) control law is used. More precisely, an ideal control law will be assumed in the form

$$
\delta \beta_{\mathrm{PID}}=-h_{P} \delta_{x}-h_{D} \delta_{x}^{\prime}-h_{I} \int_{0}^{\nu} \delta_{x} \mathrm{~d} \nu
$$

where $h_{P}, h_{D}$ and $h_{I}$ are the proportional, derivative, and integral gains, respectively. The term "ideal" is used above to emphasize that the practical implementation of the control law requires some differences to be introduced with respect to Eq. (6), as will be discussed in the next section. A stability analysis of Eq. (4) by means of Floquet's theory provides the gains necessary to stabilize a given $L_{1}$-type AEP. For example, Fig. 1 summarizes the stability region for $r_{0}=0.980$ where $\beta_{0}=0.051497$. Each isocontour line in the figure is drawn for a fixed value of $h_{I}$, and represents the lower-left boundary region of pairs $\left(h_{P}, h_{D}\right)$ for which the $L_{1}$-type AEP is stable.

To appreciate the usefulness of the PID control logic, consider a $L_{1}$-type AEP with $r_{0}=0.980$, and assume $h_{P}=h_{D}=10$. Figure 2 compares the spacecraft trajectories with and without integral control, when the reference value of the sail lightness number is $\bar{\beta}=$ $1.01 \beta_{0}$ and the injection position error is $|\delta \boldsymbol{r}| \approx 14.3 \times 10^{-5}$ (corresponding to approximately 


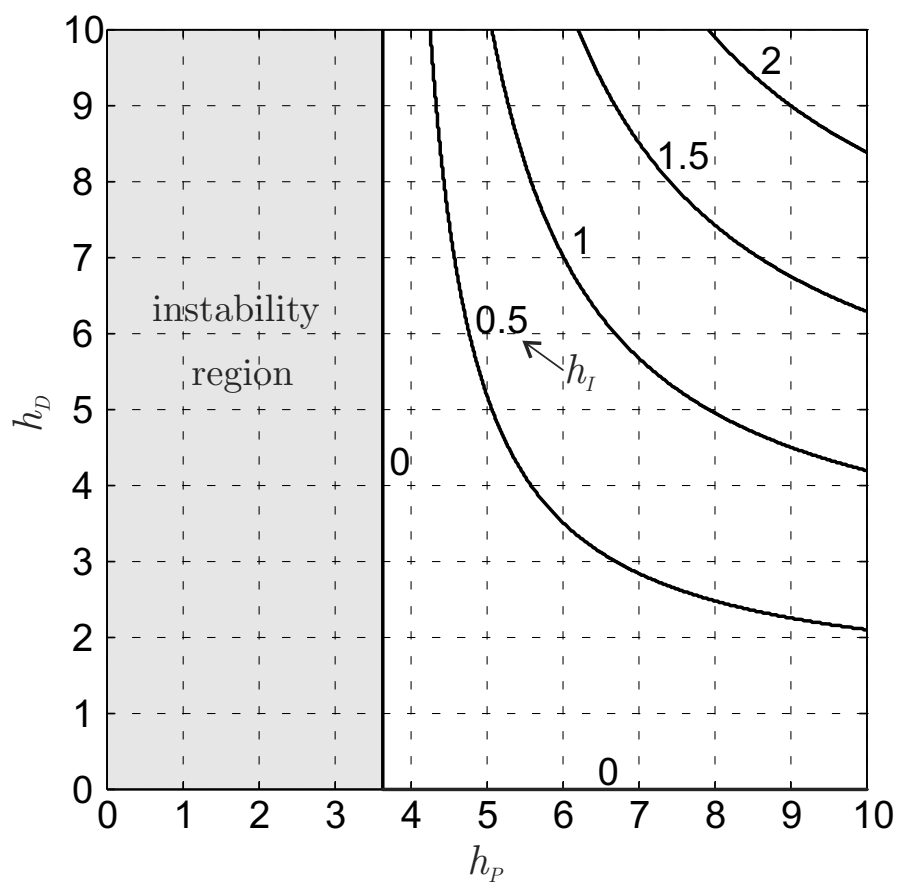

Figure 1: $\quad$ PID gains when $r_{0}=0.980$.

$20000 \mathrm{~km}$ ). Note that the integral control with $h_{I}=1$ eliminates any asymptotical error.

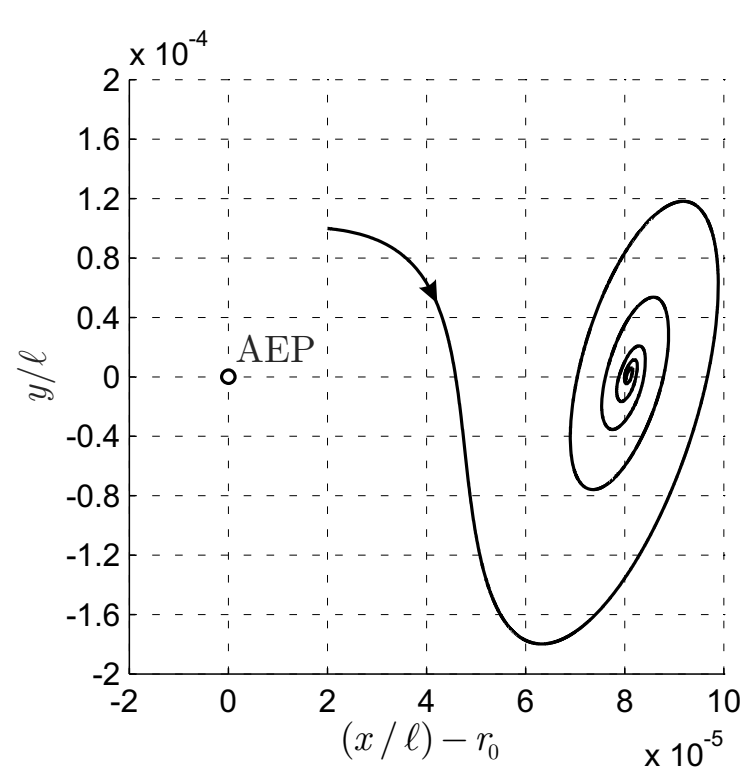

a) $\bar{\beta}=1.01 \beta_{0}$ and $h_{I}=0$

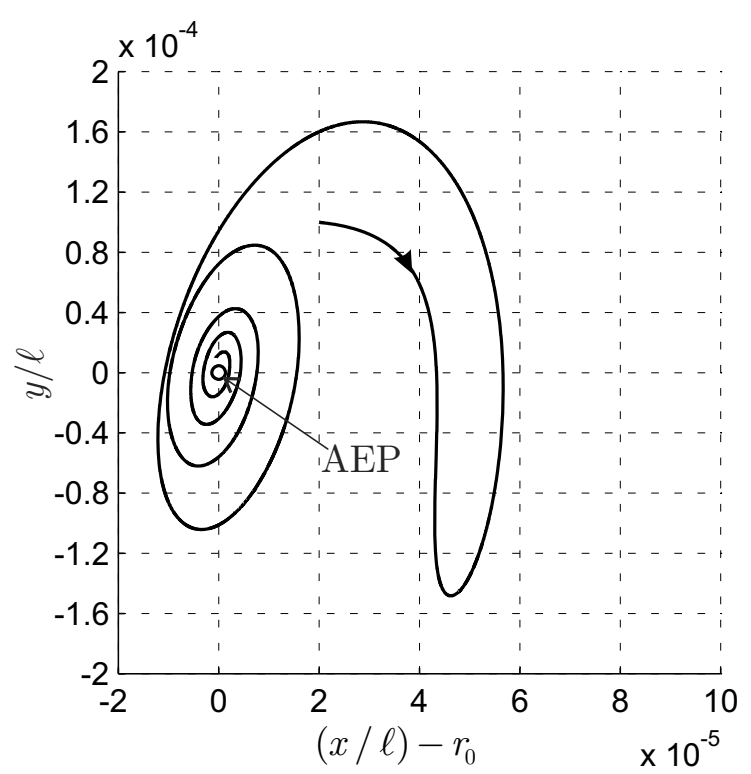

b) $\bar{\beta}=1.01 \beta_{0}$ and $h_{I}=1$

Figure 2: Effect of the integral control on the asymptotical error when $r_{0}=0.980$.

The actual implementation of a PID logic for a $\beta$-control will now be discussed assuming 
that the sail lightness number may be varied, within a specified range, by means of EMPs, whose reflectivity changes depending on the amount of voltage applied to the panels.

\section{Spacecraft mathematical model}

Consider a spacecraft of total mass $m$, whose primary propulsion system is constituted by a square, Sun-faced solar sail of total area $A$, with a slightly conical shape and whose apex is directed sunward, see Fig. 3. With such a sail configuration a radial thrust is maintained in a pure passive way [7]. Note that, in principle, the useful sail area $A$ is strictly related to the sail conical angle. However, in this simplified analysis a slight conical angle is assumed, such

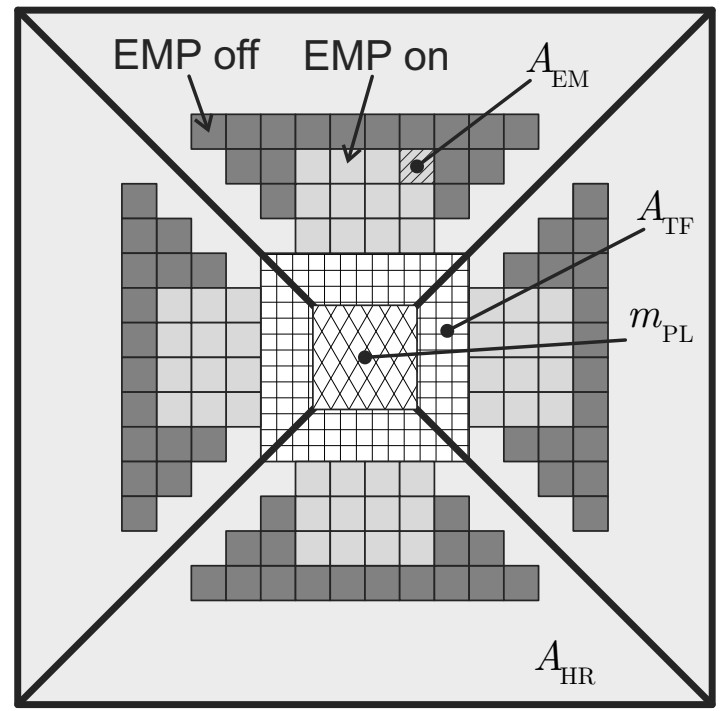

a) Front View.
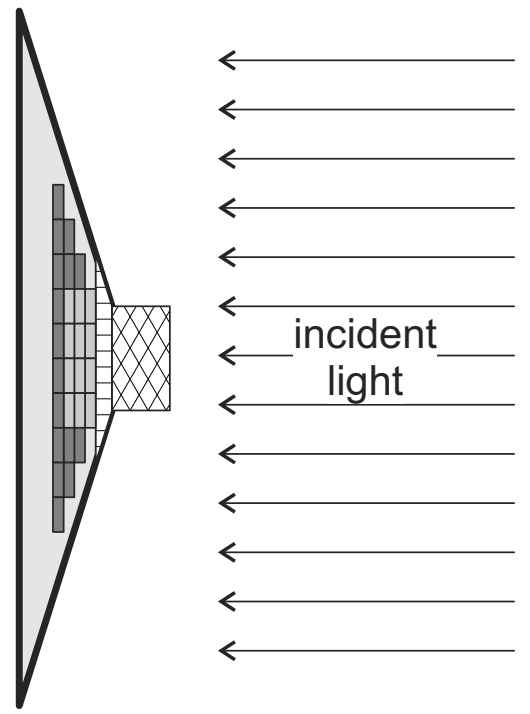

b) Side View.

Figure 3: $\quad$ Spacecraft schematic model.

The sail surface can be thought of as being constituted by three parts. The first part, of area $A_{\mathrm{HR}}$, is covered with high reflectivity material. Its main purpose is that of exploiting the solar radiation pressure to produce a propulsive thrust. A second part is covered by electrochromic material [20], which is used to modulate the thrust (within a moderate 
range) without varying the sail attitude [8]. In analogy to the design solution adopted for IKAROS $[9,10]$, this second part is constituted by $N$ EMPs. Each panel, with area $A_{\mathrm{EM}}$, varies its reflectivity as it changes its state. The latter can assume two values, either power-off (low reflectivity), or power-on state (high reflectivity), depending on the amount of voltage applied to the panel [9]. Finally, the third part, of area $A_{\mathrm{TF}}$, is covered with flexible thin-film solar cells [21], whose purpose is to guarantee the electric power required by the payload and the electrochromic material.

Each reflective surface contributes to the total spacecraft acceleration by converting, with different efficiencies, the solar radiation pressure into propulsive thrust. According to Dachwald $[22,23]$, the reflectivity of a generic sail element can be modeled by introducing an efficiency factor $\eta$. The latter is a dimensionless coefficient that can assume a value ranging from 0.5 (perfectly absorbing surface) to 1 (perfectly reflecting surface). In particular, the efficiency value can be set equal to either $\eta_{\mathrm{ON}}$ or $\eta_{\mathrm{OFF}}<\eta_{\mathrm{ON}}$ according to whether the corresponding EMP is switched on or off. The total sail's surface is written as

$$
A \triangleq A_{\mathrm{HR}}+A_{\mathrm{TF}}+N A_{\mathrm{EM}}
$$

whereas the total spacecraft mass can be expressed by adapting the simplified model described in Ref. [24], viz.

$$
m=\sigma_{\mathrm{HR}} A_{\mathrm{HR}}+\sigma_{\mathrm{TF}} A_{\mathrm{TF}}+N \sigma_{\mathrm{EM}} A_{\mathrm{EM}}+m_{\mathrm{PL}}
$$

where $m_{\mathrm{PL}}$ is the payload mass (including the spacecraft bus), and $\sigma$ is the areal density, defined as the mass per surface unit for each element. Note that $\sigma_{\mathrm{HR}}$ includes the supporting structures as, for example, booms and deployment module [24]. 


\section{Sail lightness number calculation}

Assume that, at a given time instant, a number $N_{\mathrm{ON}} \leq N$ of EMPs are switched on. To guarantee that the EMPs provide a pure thrust contribution (without introducing any additional torque on the spacecraft) it is necessary that a symmetry exists in the distribution of switched on/off panels with respect to the spacecraft's center of mass. Note that, in this simplified analysis, a failure of part of the EMPs (with a consequent loss of symmetry) is not considered. However, in that case the symmetry could be restored by simply excluding both the broken EMPs and their symmetric counterpart. This solution, of course, reduces the maximum available variation of the sail lightness number.

Let $n \geq 2$ the minimum number of EMPs that simultaneously can vary their state. For example, if $n=4$, these four panels are placed at the vertices of a rectangle whose center coincides with the spacecraft's center of mass. Observe that both $N$ and $N_{\text {ON }}$ must be integer multiples of $n$. In particular, the number $N_{\mathrm{ON}} \in \mathbb{N}$ represents the only thrust control variable, since the spacecraft thrust variation is obtained by simply setting on (or off) a suitable number of EMPs.

Because, by assumption, the mean solar sail plane is orthogonal to the direction of incoming solar rays, with the aid of Eqs. (1) and (8) the sail lightness number may be written as:

$$
\beta \triangleq \frac{\sigma^{\star}\left[\eta_{\mathrm{HR}} A_{\mathrm{HR}}+\eta_{\mathrm{TF}} A_{\mathrm{TF}}+N_{\mathrm{ON}} \eta_{\mathrm{ON}} A_{\mathrm{EM}}+\left(N-N_{\mathrm{ON}}\right) \eta_{\mathrm{OFF}} A_{\mathrm{EM}}\right]}{\left(\sigma_{\mathrm{HR}} A_{\mathrm{HR}}+\sigma_{\mathrm{TF}} A_{\mathrm{TF}}+N \sigma_{\mathrm{EM}} A_{\mathrm{EM}}+m_{\mathrm{PL}}\right)}
$$

where $\sigma^{\star} \simeq 1.53 \mathrm{~g} / \mathrm{m}^{2}$ is the critical sail loading parameter [25]. The presence of EMPs is useful for varying the sail lightness number between a minimum value $\beta_{\min }$, when all of EMPs are switched off $\left(N_{\mathrm{ON}}=0\right)$ and a maximum value $\beta_{\max }$, when $N_{\mathrm{ON}}=N$. From Eq. $(9), \beta_{\min }$ 
and $\beta_{\max }$ are given by:

$$
\begin{aligned}
\beta_{\mathrm{min}} & =\frac{\sigma^{\star}\left[\eta_{\mathrm{HR}} A_{\mathrm{HR}}+\eta_{\mathrm{TF}} A_{\mathrm{TF}}+N \eta_{\mathrm{OFF}} A_{\mathrm{EM}}\right]}{\left(\sigma_{\mathrm{HR}} A_{\mathrm{HR}}+\sigma_{\mathrm{TF}} A_{\mathrm{TF}}+N \sigma_{\mathrm{EM}} A_{\mathrm{EM}}+m_{\mathrm{PL}}\right)} \\
\beta_{\max } & =\frac{\sigma^{\star}\left[\eta_{\mathrm{HR}} A_{\mathrm{HR}}+\eta_{\mathrm{TF}} A_{\mathrm{TF}}+N \eta_{\mathrm{ON}} A_{\mathrm{EM}}\right]}{\left(\sigma_{\mathrm{HR}} A_{\mathrm{HR}}+\sigma_{\mathrm{TF}} A_{\mathrm{TF}}+N \sigma_{\mathrm{EM}} A_{\mathrm{EM}}+m_{\mathrm{PL}}\right)}
\end{aligned}
$$

Note that $\beta$ can only take a finite number of values (equal to $N / n+1$ ) within its variation interval. Therefore $\beta$ is actually chosen from a "thrust setting table", similar to what happens for solar electric propulsion systems [26].

The mean sail lightness number $\bar{\beta} \triangleq\left(\beta_{\min }+\beta_{\max }\right) / 2$ is obtained when one half of EMPs are switched on, that is, when $N_{\mathrm{ON}}=N / 2$. The quantity $\bar{\beta}$ can be thought of as a reference sail lightness number and its value is chosen to be as close as possible to $\beta_{0}$ given by Eq. (3).

The maximum allowable variation with respect to the mean value will be referred to as $\Delta \beta \triangleq\left(\beta_{\max }-\beta_{\min }\right) / 2$ and represents an index of the maximum spacecraft capability of varying its performance during the mission. Combining Eqs. (10) and (11) it is found that

$$
\begin{aligned}
\bar{\beta} & =\frac{\sigma^{\star}\left[2 \eta_{\mathrm{HR}} A_{\mathrm{HR}}+2 \eta_{\mathrm{TF}} A_{\mathrm{TF}}+N\left(\eta_{\mathrm{ON}}+\eta_{\mathrm{OFF}}\right) A_{\mathrm{EM}}\right]}{2\left(\sigma_{\mathrm{HR}} A_{\mathrm{HR}}+\sigma_{\mathrm{TF}} A_{\mathrm{TF}}+N \sigma_{\mathrm{EM}} A_{\mathrm{EM}}+m_{\mathrm{PL}}\right)} \\
\Delta \beta & =\frac{\sigma^{\star} N\left(\eta_{\mathrm{ON}}-\eta_{\mathrm{OFF}}\right) A_{\mathrm{EM}}}{2\left(\sigma_{\mathrm{HR}} A_{\mathrm{HR}}+\sigma_{\mathrm{TF}} A_{\mathrm{TF}}+N \sigma_{\mathrm{EM}} A_{\mathrm{EM}}+m_{\mathrm{PL}}\right)}
\end{aligned}
$$

The term $A_{\mathrm{TF}}$ in the previous equations will now be expressed as a function of $m_{\mathrm{PL}}$ and $A_{\mathrm{EM}}$. This is possible by observing that the surface covered with flexible thin-film solar cells must generate an electric power sufficient for supplying power to both the payload and the EMPs. More precisely, introduce a payload specific power $\alpha_{\mathrm{PL}}$, defined as the electric power per unit mass required by the payload, and a solar array efficiency $\epsilon_{\mathrm{TF}}$ [27], which coincides with the ratio between the solar arrays output electric power per unit area and the local 
solar irradiance.

The power generation system is designed in a conservative way under the following assumptions: 1) end life conditions (when the degradation effects are maximum), 2) maximum solar distance during the mission (when the solar irradiance is minimum), and 3) maximum required power condition. Assuming that the degradation effects are all included in a single coefficient $\epsilon_{\mathrm{TF}}$ and that the maximum Sun-spacecraft distance is equal to one Astronomical Unit, a simplified electric power balance between the power generated by the solar cells and that absorbed by EMPs and payload provides the required value of $A_{\mathrm{TF}}$ :

$$
A_{\mathrm{TF}}=\frac{\phi_{\mathrm{EM}} N A_{\mathrm{EM}}+\alpha_{\mathrm{PL}} m_{\mathrm{PL}}}{\epsilon_{\mathrm{TF}} W_{\oplus}}
$$

where $W_{\oplus} \triangleq 1366 \mathrm{~W} / \mathrm{m}^{2}$ is the solar constant, and $\phi_{\mathrm{EM}}$ is the electric power per unit area required by the electrochromic material.

\section{Spacecraft sizing}

When Eq. (14) is substituted into Eq. (9) and the solar sail's physical characteristics are fixed, $\beta$ is shown to depend linearly on the control variable $N_{\mathrm{ON}} \in\{0, n, 2 n, \ldots, N\}$, that is

$$
\beta=\beta_{\mathrm{min}}+k_{\beta} N_{\mathrm{ON}} \quad \text { with } \quad k_{\beta} \triangleq \frac{\sigma^{\star} A_{\mathrm{EM}}\left(\eta_{\mathrm{ON}}-\eta_{\mathrm{OFF}}\right)}{\sigma_{\mathrm{HR}} A_{\mathrm{HR}}+\sigma_{\mathrm{TF}} A_{\mathrm{TF}}+N \sigma_{\mathrm{EM}} A_{\mathrm{EM}}+m_{\mathrm{PL}}}
$$

The sail lightness number also depends, in a more involved way, on the four design parameters $N, m_{\mathrm{PL}}, A_{\mathrm{HR}}$, and $A_{\mathrm{EM}}$. However, by means of Eqs. (12) and (13), $N$ and $A_{\mathrm{HR}}$ are more conveniently expressed as a function of two other mission parameters, that is, $\beta_{0}$ and $\Delta \beta$. 
Indeed, assuming that $\bar{\beta}=\beta_{0}$, the result is

$$
\begin{aligned}
N & =n\left\|\left(\frac{m_{\mathrm{PL}} / A_{\mathrm{EM}}}{n \sigma^{\star}}\right) \frac{\Delta \beta}{c_{1} \beta_{0}+c_{2} \Delta \beta+c_{3}}\right\| \\
A_{\mathrm{HR}} & =\frac{m_{\mathrm{PL}}}{\sigma^{\star}}\left(\frac{c_{4}}{c_{1} \beta_{0}+c_{2} \Delta \beta+c_{3}}-c_{6}\right)-c_{5} N A_{\mathrm{EM}}
\end{aligned}
$$

where $\|\cdot\|$ is the round function, which is introduced in Eq. (16) because $N$ can only take integer values. Also $c_{i}$ (with $i=1,2, \ldots, 6$ ) are dimensionless coefficients, independent of the design parameters, defined as:

$$
\begin{gathered}
c_{1} \triangleq \frac{\epsilon_{\mathrm{TF}} W_{\oplus}\left(\eta_{\mathrm{ON}}-\eta_{\mathrm{OFF}}\right)\left(\sigma_{\mathrm{HR}} / \sigma^{\star}\right)}{2\left(\sigma_{\mathrm{HR}} \eta_{\mathrm{TF}} \alpha_{\mathrm{PL}}-\sigma_{\mathrm{TF}} \alpha_{\mathrm{PL}} \eta_{\mathrm{HR}}-\epsilon_{\mathrm{TF}} W_{\oplus} \eta_{\mathrm{HR}}\right)} \\
c_{2} \triangleq \frac{2 \sigma_{\mathrm{EM}} \epsilon_{\mathrm{TF}} W_{\oplus} \eta_{\mathrm{HR}}+2 \phi_{\mathrm{EM}}\left(\sigma_{\mathrm{TF}} \eta_{\mathrm{HR}}-\sigma_{\mathrm{HR}} \eta_{\mathrm{TF}}\right)-\sigma_{\mathrm{HR}} \epsilon_{\mathrm{TF}} W_{\oplus}\left(\eta_{\mathrm{ON}}+\eta_{\mathrm{OFF}}\right)}{2 \sigma^{\star}\left(\sigma_{\mathrm{HR}} \eta_{\mathrm{TF}} \alpha_{\mathrm{PL}}-\sigma_{\mathrm{TF}} \alpha_{\mathrm{PL}} \eta_{\mathrm{HR}}-\epsilon_{\mathrm{TF}} W_{\oplus} \eta_{\mathrm{HR}}\right)} \\
c_{3} \triangleq \frac{-\eta_{\mathrm{HR}} \epsilon_{\mathrm{TF}} W_{\oplus}\left(\eta_{\mathrm{ON}}-\eta_{\mathrm{OFF}}\right)}{2\left(\sigma_{\mathrm{HR}} \eta_{\mathrm{TF}} \alpha_{\mathrm{PL}}-\sigma_{\mathrm{TF}} \alpha_{\mathrm{PL}} \eta_{\mathrm{HR}}-\epsilon_{\mathrm{TF}} W_{\oplus} \eta_{\mathrm{HR}}\right)} \\
c_{4} \triangleq \frac{\sigma^{\star}\left(\eta_{\mathrm{ON}}-\eta_{\mathrm{OFF}}\right)}{2 \sigma_{\mathrm{HR}}} \\
c_{5} \triangleq \frac{\sigma_{\mathrm{EM}}}{\sigma_{\mathrm{HR}}}+\frac{\sigma_{\mathrm{TF}} \phi_{\mathrm{EM}}}{\sigma_{\mathrm{HR}} \epsilon_{\mathrm{TF}} W_{\oplus}} \\
c_{6} \triangleq \frac{\sigma^{\star}}{\sigma_{\mathrm{HR}}}\left(\frac{\sigma_{\mathrm{TF}} \alpha_{\mathrm{PL}}}{\epsilon_{\mathrm{TF}} W_{\oplus}}+1\right)
\end{gathered}
$$

Note that, as a consequence of the discretization process induced by the finite number of EMPs in Eq. (16), the attainable value of $\bar{\beta}$ is actually different from the required value $\beta_{0}$. This confirms the importance of an integral action in the control law (6). For a given mission scenario (that is, $\beta_{0}$ and $\Delta \beta$ are given) and for a prescribed set of coefficients $c_{i}$, 
Eqs. (16)-(17) can be used to estimate the required values of $N$ and $A_{\mathrm{HR}}$ as a function of the payload mass $m_{\mathrm{PL}}$ and the area $A_{\mathrm{EM}}$ of a single panel. It is worth noting that in the limiting case in which $\Delta \beta=0$ (when the sail is unable to perform a $\beta$-control) Eq. (16) states that, as expected, $N=0$. Moreover, combining Eq. (17) with (18), (20), and (21), it can be shown that when $\Delta \beta=0, A_{\mathrm{HR}}$ is independent of the optical characteristics of the electrochromic material.

To summarize, for a given set of data $\beta_{0}, \Delta \beta, m_{\mathrm{PL}}, A_{\mathrm{EM}}$, and $n$, the value of $N$ is calculated from Eq. (16), $A_{\mathrm{HR}}$ from Eq. (17), $A_{\mathrm{TF}}$ from Eq. (14), $A$ from Eq. (7), $m$ from Eq. (8), and, finally, $\beta_{\min }$ and $\beta_{\max }$ from Eqs. (10)-(11).

\section{Control law implementation}

From the previous discussion, the sail lightness number can be effectively controlled, through a PID control logic, in the interval $\beta \in\left[\beta_{\min }, \beta_{\max }\right]$ according to Eq. (15). However, unlike the ideal steering law described by Eq. (6), two other aspects must be taken into account. In fact, the propulsive acceleration is both subjected to a discretization effect due to the presence of a finite number of EMPs, and to a possible control saturation. In particular, the quantization error introduced by the discretization process is $n k_{\beta}$, which corresponds to the minimum sail lightness number variation obtained when a single group of $n$ EMPs changes its state. On the other hand, a saturation occurs (and a windup behavior takes place) when the EMPs are unable to provide the sail lightness number variation $\delta \beta_{\text {PID }}$ commanded by the control logic described in Eq. (6). This phenomenon can have a fundamental influence on the behavior of the controlled system and, in some cases, it can cause instability.

To effectively counteract the saturation effect, an anti-windup compensator is therefore introduced. Its main purpose is to reduce the integral action when the $\beta$-control saturates [28]. A block diagram for the $\beta$-control logic, including the anti-windup compensator (with a gain $h_{\mathrm{AW}} \geq 0$ ), is shown in Fig. 4. Recall that $\bar{\beta}$ is the reference sail lightness number. If the 
current spacecraft position $(x / \ell)$ does not coincide with the desired AEP, an error $\delta_{x} \neq 0$ occurs. This error signal is processed by the PID block whose output is first translated into a (discrete) lightness number variation, and then compared to the minimum/maximum obtainable values. When a saturation takes place, the integral of the difference between the lightness value required by the control logic and the saturated value is integrated by the anti-windup block and the result is eventually added to the PID output. Note that the contribution from the anti-windup block is different from zero only when the control system is saturated, that is, when $\delta \beta= \pm k_{\beta} N / 2$.

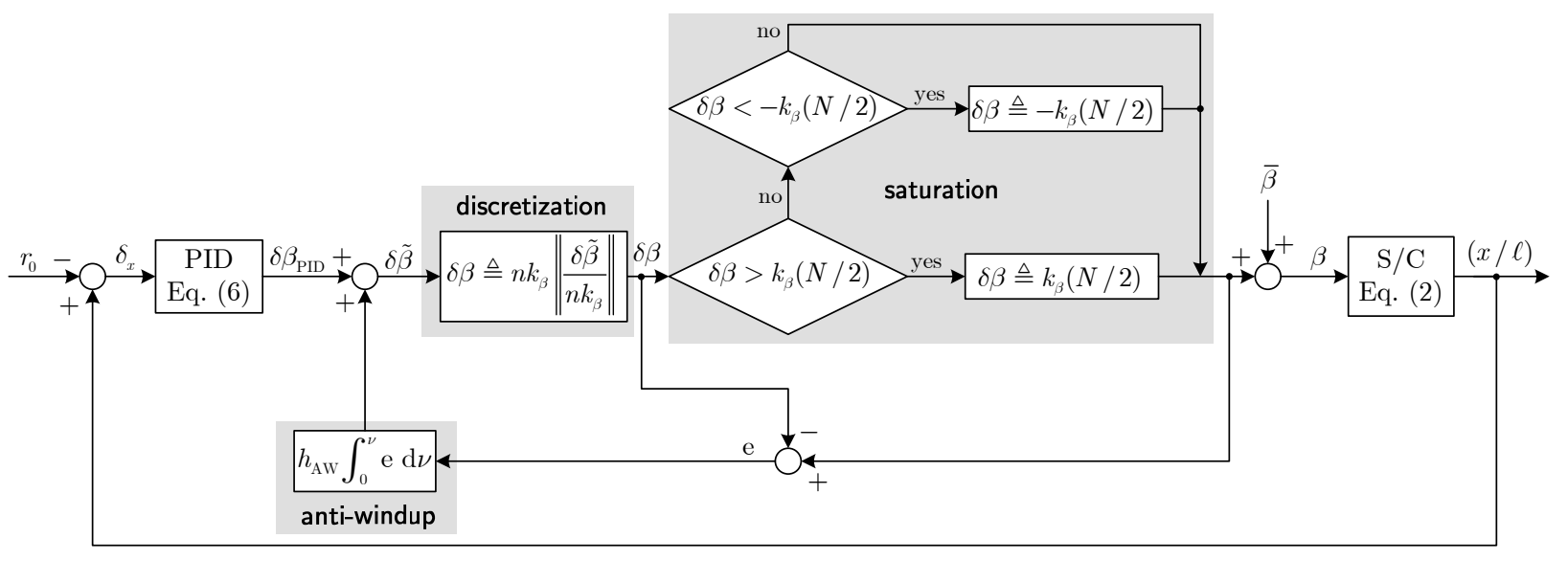

Figure 4: $\quad$ Block diagram for the $\beta$-control logic.

\section{Numerical Simulations}

The previous mathematical model will now be used to illustrate the preliminary design of a solar sail spacecraft, whose mission requirement is to maintain an $L_{1}$-type AEP $[14,29]$ in the neighborhood of a classical $L_{1}$ Lagrange point in the Sun-(Earth+Moon) system. Within this mission scenario the AEP position is, at any time instant, along the Sun-Earth line at a dimensionless distance $r_{0}=0.98$ from the center of mass $C$.

Some spacecraft characteristics, as for example a payload mass of $91 \mathrm{~kg}$, have been extrapolated from the Heliostorm Warning Mission (a variant of Geostorm Warning Mission [2]), 
using tables and plots taken from Ref. [30]. For the high-reflectivity element, a film with an aluminum-coated front side and a chromium-coated back side is assumed [23]. As far as the electrochromic part is concerned, $A_{\mathrm{EM}}=1 \mathrm{~m}^{2}$ and $n=8$ are assumed. Moreover, according to Ref. [8], and in the absence of additional data, $\eta_{\mathrm{ON}}$ is taken equal to $\eta_{\mathrm{HR}}$ and $\eta_{\mathrm{OFF}}=0.5$, which corresponds to a complete absorption of the incoming photons. Finally, thin-film solar cells with $\mathrm{Cu}(\mathrm{In}, \mathrm{Ga}) \mathrm{Se} 2$ as an absorber layer and Kapton as a substrate are assumed $[21,27]$. A conservatively small value of $\epsilon_{\mathrm{TF}}=10 \%$ is used to model the effects of a performance degradation with time. The physical data necessary to calculate the coefficients $c_{i}$ in Eqs. (18)-(23) are summarized in Table 1, along with the corresponding bibliographical information from which those data have been derived. The values of the six coefficients are therefore: $c_{1}=-0.8303, c_{2}=-56.5111, c_{3}=0.2031, c_{4}=0.055, c_{5}=16.1467$, and $c_{6}=0.2706$.

\begin{tabular}{cccc}
\hline \hline parameter & value & units & Ref. \\
\hline$\alpha_{\mathrm{PL}}$ & 8 & $\mathrm{~W} / \mathrm{kg}$ & {$[30]$} \\
$\sigma_{\mathrm{HR}}$ & 5.68 & $\mathrm{~g} / \mathrm{m}^{2}$ & {$[30]$} \\
$\sigma_{\mathrm{EM}}$ & 80 & $\mathrm{~g} / \mathrm{m}^{2}$ & {$[31]$} \\
$\sigma_{\mathrm{TF}}$ & 80 & $\mathrm{~g} / \mathrm{m}^{2}$ & {$[27]$} \\
$\eta_{\mathrm{ON}}$ & 0.908 & & \\
$\eta_{\mathrm{OFF}}$ & 0.5 & & {$[8]$} \\
$\eta_{\mathrm{HR}}$ & 0.908 & & {$[23]$} \\
$\eta_{\mathrm{TF}}$ & 0.5 & & \\
$\phi_{\mathrm{EM}}$ & 20 & $\mathrm{~W} / \mathrm{m}^{2}$ & \\
$\epsilon_{\mathrm{TF}}$ & 0.1 & & {$[27]$} \\
\hline \hline
\end{tabular}

Table 1: Physical reference data.

The maximum allowable variation $\Delta \beta$ must be chosen depending on the estimated perturbations magnitude that will affect the spacecraft dynamics at the desired AEP. The solar sail characteristic parameters, obtained with the methodology outlined above, have been 
summarized in Table 2 for $\Delta \beta / \beta_{0}=\{1 \%, 2 \%, 3 \%, 4 \%\}$. The table shows a rapid growth of $N$ with an increase of $\Delta \beta / \beta_{0}$, which implies an increase of the sail side (proportional to $\sqrt{A})$.

\begin{tabular}{c|c|c|c|c|c}
\hline \hline \multirow{2}{*}{ parameter } & \multicolumn{4}{|c|}{$\Delta \beta / \beta_{0}$} & Eq. \\
& $1 \%$ & $2 \%$ & $3 \%$ & $4 \%$ & $\#$ \\
\hline$N$ & 232 & 600 & 1256 & 2792 & $(16)$ \\
$A_{\mathrm{HR}}\left[\mathrm{m}^{2}\right]$ & 5064 & 6219.3 & 8381.6 & 13236.7 & $(17)$ \\
$A_{\mathrm{TF}}\left[\mathrm{m}^{2}\right]$ & 39.3 & 93.2 & 189.2 & 414.1 & $(14)$ \\
$A\left[\mathrm{~m}^{2}\right]$ & 5335.3 & 6912.5 & 9826.8 & 16442.8 & $(7)$ \\
$m[\mathrm{~kg}]$ & 141.5 & 181.8 & 254.2 & 422.7 & $(8)$ \\
$\beta_{\min } \times 10^{2}$ & 5.119646 & 5.044816 & 5.015082 & 4.930915 & $(10)$ \\
$\beta_{\max } \times 10^{2}$ & 5.222018 & 5.250858 & 5.323488 & 5.343261 & $(11)$ \\
$\bar{\beta} \times 10^{2}$ & 5.170832 & 5.147837 & 5.169285 & 5.137088 & \\
$n k_{\beta} \times 10^{5}$ & 3.530091 & 2.747230 & 1.964368 & 1.181506 & \\
$k_{\beta} \times 10^{6}$ & 4.412614 & 3.434037 & 2.45546 & 1.476883 & $(15)$ \\
\hline \hline
\end{tabular}

Table 2: $\quad$ Spacecraft parameters for $r_{0}=0.980$ and $\beta_{0}=0.051497$.

For illustrative purposes assume that $\Delta \beta / \beta_{0}=1 \%$ and use the spacecraft parameters from Table 2. To discuss the $\beta$-control effectiveness, two cases have been considered, with and without $\left(h_{\mathrm{AW}}=0\right)$ the effect of the anti-windup logic. In both cases the PID parameters are chosen to be $h_{P}=10, h_{D}=10$, and $h_{I}=1$ (this corresponds to a stable configuration, see Fig. 1). An initial spacecraft position error of $20000 \mathrm{~km}$ and a velocity error of $150 \mathrm{~m} / \mathrm{s}$ has been simulated.

Firstly, the anti-windup gain $h_{\mathrm{AW}}$ is set equal to zero, and the simulation results are shown in Figs. 5(a) and 6(a). Due to a control system saturation, the number of switched on EMPs is either $N_{\mathrm{ON}}=0$ or $N_{\mathrm{ON}}=232$, and the control system is unable to drive the spacecraft toward the desired $L_{1}$-type AEP.

However, when the anti-windup controller is engaged, with $h_{\mathrm{AW}}=10$, the sail lightness 


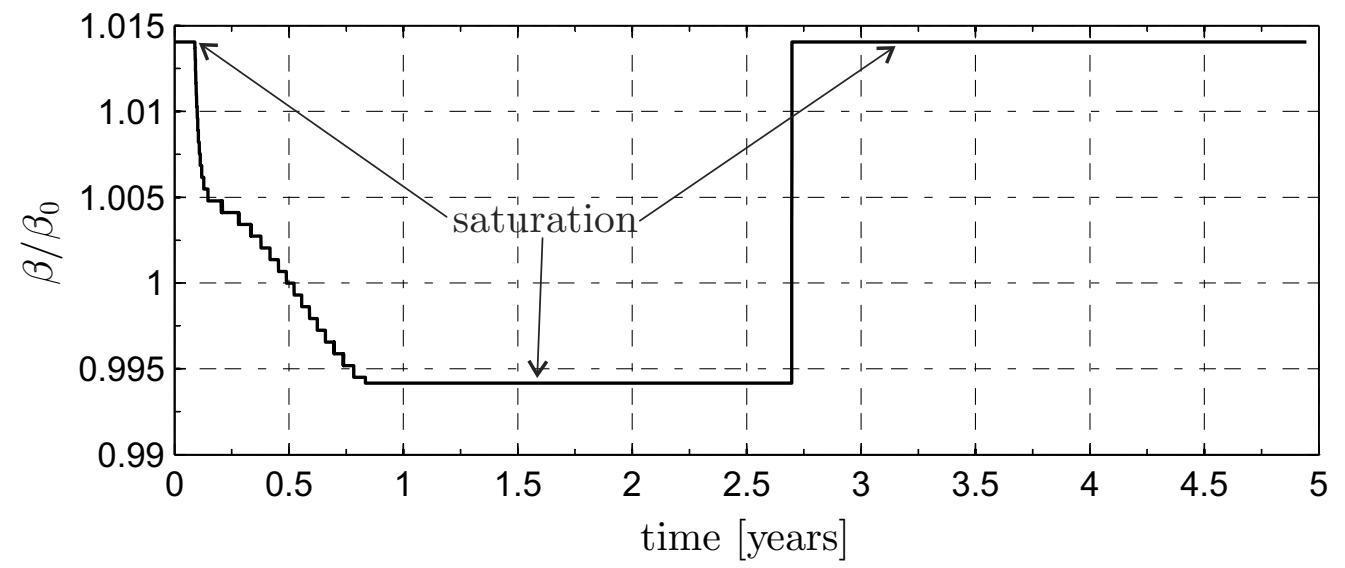

a) Without anti-windup filter $\left(h_{\mathrm{AW}}=0\right)$.

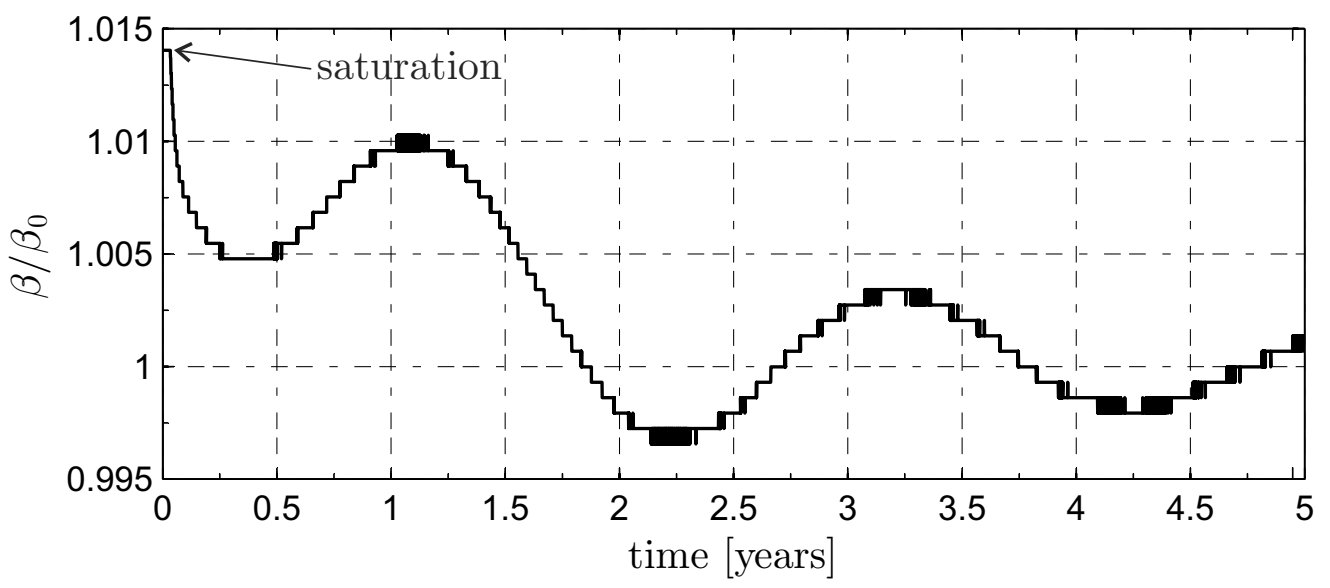

b) With anti-windup filter $\left(h_{\mathrm{AW}}=10\right)$.

Figure 5: Sail lightness number variation time response for $r_{0}=0.980$ and $\beta_{0}=$ 0.051497 .

number is quickly steered within its linear variation range (see Fig. 5(b)), and the spacecraft dynamics remains bounded around the desired $L_{1}$-type AEP (see Fig. 6(b)).

\section{Conclusions}

Electrochromic material panels can be effectively used as a simple control means to stabilize a solar sail with a fixed attitude about an artificial, collinear, Lagrange point. The panels are used to vary the sail lightness number by changing their state from on to off or off to on in such a way to provide a propulsive acceleration variation without introducing 


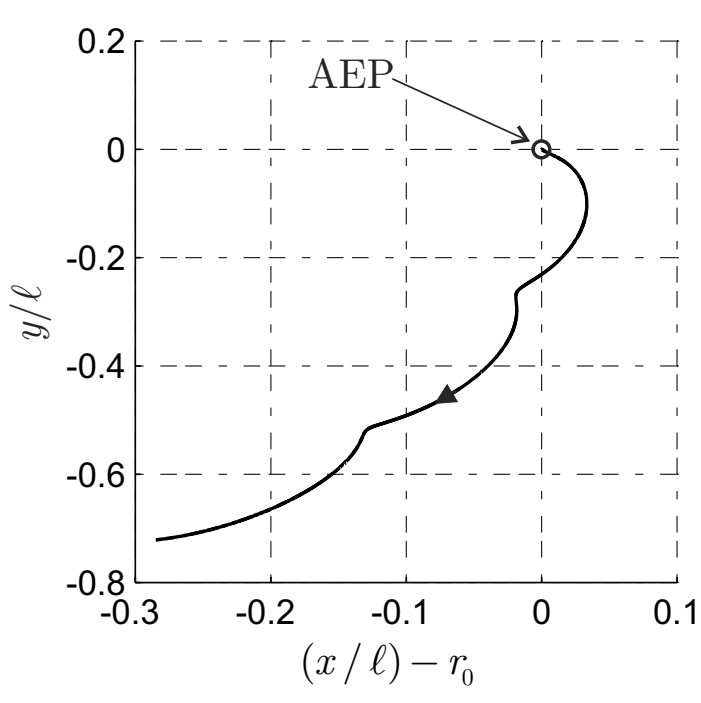

a) Without anti-windup filter $\left(h_{\mathrm{AW}}=0\right)$.

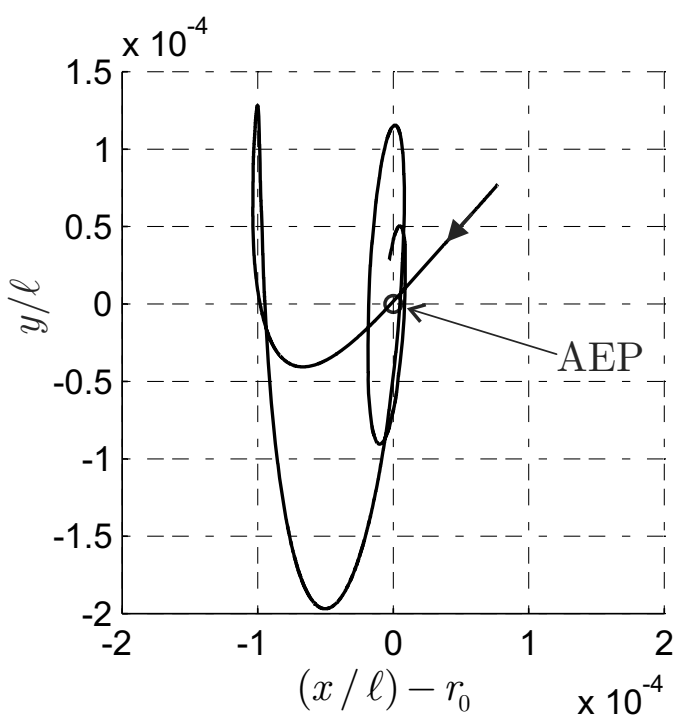

b) With anti-windup filter $\left(h_{\mathrm{AW}}=10\right)$.

Figure 6: Spacecraft trajectory in the rotating-pulsating frame $\mathcal{T}$ for $r_{0}=0.980$ and $\beta_{0}=0.051497$.

additional torques on the spacecraft. A simplified mathematical model has been developed to define the main spacecraft parameters, including the sail dimension and the total spacecraft mass, as a function of the desired artificial equilibrium point position. Finally, a simple steering logic, in the form of a proportional-integral-derivative control system, has been introduced for stabilizing the spacecraft about a $L_{1}$-type point.

A natural extension of the analysis discussed in this Note is to explore the influence of the sail conical shape on both the control torque and the variation in sail lightness number.

\section{References}

[1] McKay, R. J., Macdonald, M., Biggs, J., and McInnes, C. R., "Survey of Highly NonKeplerian Orbits with Low-Thrust Propulsion," Journal of Guidance, Control and Dynamics, Vol. 34, No. 3, May-June 2011, pp. 645-666. doi: 10.2514/1.52133.

[2] West, J. L., "The Geostorm Warning Mission: enhanced opportunities based on new technol- 
ogy," 14th AAS/AIAA Space Flight Mechanics Conference, Maui, HI, February 8-12 2004, Paper AAS 04-102.

[3] McInnes, C. R., "Minimum mass solar shield for terrestrial climate control," Journal of the British Interplanetary Society, Vol. 55, 2002, pp. 307-311.

[4] Biggs, J. D. and McInnes, C. R., "Passive Orbit Control for Space-Based Geo-Engineering," Journal of Guidance, Control and Dynamics, Vol. 33, No. 3, May-June 2010, pp. 1017-1020. doi: $10.2514 / 1.46054$.

[5] McInnes, C. R., McDonald, A. J. C., Simmons, J. F. L., and MacDonald, E. W., "Solar Sail Parking in Restricted Three-Body Systems," Journal of Guidance, Control, and Dynamics, Vol. 17, No. 2, March-April 1994, pp. 399-406. doi: 10.2514/3.21211.

[6] Bookless, J. and McInnes, C. R., "Control of Lagrange point orbits using solar sail propulsion," Acta Astronautica, Vol. 62, No. 1-2, January-February 2008, pp. 159-176. doi: 10.1016/j.actaastro.2006.12.051.

[7] McInnes, C. R., "Passive Control of Displaced Solar Sail Orbits," Journal of Guidance, Control and Dynamics, Vol. 21, No. 6, November-December 1998, pp. 975-982. doi: $10.2514 / 2.4334$.

[8] Lücking, C. M., Colombo, C., and McInnes, C. R., "Orbit control of high area-to-mass ratio spacecraft using electrochromic coating," 61st International Astronautical Congress, Prague, Czech Republic, September 27 - October 1 2010, Paper IAC-10-C1.2.7.

[9] Mori, O., Tsuda, Y., Shirasawa, Y., Saiki, T., Mimasu, Y., and Kawaguchi, J., "Attitude Control of IKAROS Solar Sail Spacecraft and Its Flight Results," 61st International Astronautical Congress, Prague, Czech Republic, September 27-October 1 2010, Paper IAC-10.C1.4.3.

[10] Funase, R., Shirasawa, Y., Mimasu, Y., Mori, O., Tsuda, Y., Saiki, T., and Kawaguchi, J., "Fuel-free and Oscillation-free Attitude Control of IKAROS Solar Sail Spacecraft Using Re- 
flectivity Control Device," 28th International Symposium on Space Technology and Science, Okinawa, Japan, June 5-12 2011.

[11] Szebehely, V., Theory of orbits: the restricted problem of three bodies, Academic Press Inc., 1967, pp. 255, 587-602.

[12] Luzum, B., Capitaine, N., Fienga, A., Folkner, W., Fukushima, T., Hilton, J., Hohenkerk, C., Krasinsky, G., Petit, G., Pitjeva, E., Soffel, M., and Wallace, P., "The IAU 2009 system of astronomical constants: the report of the IAU working group on numerical standards for Fundamental Astronomy." Celestial Mechanics and Dynamical Astronomy, Vol. 110, No. 4, August 2011, pp. 293-304. doi: 10.1007/s10569-011-9352-4.

[13] Quarta, A. A. and Mengali, G., "Optimal Switching Strategy for Radially Accelerated Trajectories," Celestial Mechanics and Dynamical Astronomy, Vol. 105, No. 4, December 2009, pp. 361-377. doi: 10.1007/s10569-009-9233-2.

[14] Baoyin, H. and McInnes, C. R., "Solar Sail Equilibria in the Elliptical Restricted ThreeBody Problem," Journal of Guidance, Control, and Dynamics, Vol. 29, No. 3, May-June 2006, pp. 538-543. doi: 10.2514/1.15596.

[15] Aliasi, G., Mengali, G., and Quarta, A. A., "Artificial Equilibrium Points for a Generalized Sail in the Circular Restricted Three-Body Problem," Celestial Mechanics and Dynamical Astronomy, Vol. 110, No. 4, August 2011, pp. 343-368. doi: 10.1007/s10569-011-9366-y.

[16] Aliasi, G., Mengali, G., and Quarta, A. A., "Artificial Equilibrium Points for a Generalized Sail in the Elliptic Restricted Three-Body Problem," Celestial Mechanics and Dynamical Astronomy, 2012 (in press). doi: 10.1007/s10569-012-9425-z.

[17] Biggs, J. D., McInnes, C. R., and Waters, T., "Control of Solar Sail Periodic Orbits in the Elliptic Three-Body Problem," Journal of Guidance, Control and Dynamics, Vol. 32, No. 1, January-February 2009, pp. 318-320. doi: 10.2514/1.38362. 
[18] Bolle, A. and Circi, C., "Solar Sail Attitude Control Through In-Plane Moving Masses," Proceedings of the Institution of Mechanical Engineers, Part G: Journal of Aerospace Engineering, Vol. 222, No. 1, January 2008, pp. 81-94. doi: 10.1243/09544100JAERO223.

[19] Circi, C., "Three-Axis Attitude Control Using Combined Gravity-Gradient and Solar Pressure," Proceedings of the Institution of Mechanical Engineers, Part G: Journal of Aerospace Engineering, Vol. 221, No. 1, January 2007, pp. 85-90. doi: 10.1243/09544100JAERO48.

[20] Kislov, N., "Variable Reflectance/Transmittance Coatings for Solar Sail Altitude Control and Three Axis Stabilization," AIP Conference Proceedings, Vol. 699, No. 1, 2004, pp. 103-111. doi: $10.1063 / 1.1649563$.

[21] Chopra, K. L., Paulson, P. D., and Dutta, V., "Thin-film solar cells: an overview," Progress in Photovoltaics: Research and Applications, Vol. 12, No. 2-3, March-May 2004, pp. 69-92. doi: $10.1002 /$ pip.541.

[22] Dachwald, B., "Optimization of Interplanetary Solar Sailcraft Trajectories Using Evolutionary Neurocontrol," Journal of Guidance, Control, and Dynamics, Vol. 27, No. 1, JanuaryFebruary 2004, pp. 66-72. doi: 10.2514/1.9286.

[23] Dachwald, B., "Interplanetary Mission Analysis for Non-Perfectly Reflecting Solar Sailcraft Using Evolutionary Neurocontrol," AAS/AIAA Astrodynamics Specialist Conference, Big Sky, Montana, August 3-7 2003, Paper AAS 03-579.

[24] Baig, S. and McInnes, C. R., "Artificial Three-Body Equilibria for Hybrid Low-Thrust Propulsion," Journal of Guidance, Control and Dynamics, Vol. 31, No. 6, NovemberDecember 2008, pp. 1644-1655. doi: 10.2514/1.36125.

[25] McInnes, C. R., Solar Sailing: Technology, Dynamics and Mission Applications, chap. 2, Springer-Praxis Series in Space Science and Technology, Springer-Verlag, 1999, pp. 32-55, ISBN: 1-852-33102-X. 
[26] Quarta, A. A. and Mengali, G., "Minimum-Time Space Missions with Solar Electric Propulsion," Aerospace Science and Technology, Vol. 15, No. 5, July-August 2011, pp. 381-392. doi: 10.1016/j.ast.2010.09.003.

[27] Otte, K., Makhova, L., Braun, A., and Konovalov, I., "Flexible Cu(In,Ga)Se ${ }_{2}$ thin-film solar cells for space application," Thin Solid Films, Vol. 511-512, July 2006, pp. 613-622. doi: 10.1016/j.tsf.2005.11.068.

[28] Franklin, G. F., Powell, J. D., and Emami-Naeini, A., Feedback Control of Dynamic Systems, Prentice-Hall, 4th ed., 2002, p. 228, ISBN: 0-130-32393-4.

[29] McInnes, C. R., "Artificial Lagrange Points for a Partially Reflecting Flat Solar Sail," Journal of Guidance, Control and Dynamics, Vol. 22, No. 1, January-February 1999, pp. 185-187. doi: $10.2514 / 2.7627$.

[30] Young, R. M., "Updated Heliostorm Warning Mission: Enhancements Based on New Technology," 48th AIAA/ASME/ASCE/AHS/ASC Structures, Structural Dynamics, and Materials Conference, Honolulu, Hawaii, April 23-26 2007, Paper AIAA 2007-2249.

[31] Adeli, S. N., Lappas, V., and Wie, B., "A scalable bus-based attitude control system for Solar Sails," Advances in Space Research, Vol. 48, No. 11, December 2011, pp. 1836-1847. doi: 10.1016/j.asr.2011.08.024. 